\title{
The diagnostic and prognostic value of nuclear matrix protein 22 in bladder cancer
}

\author{
Xingxing Tang ${ }^{\#}$, Yudong Cao ${ }^{\#}$, Jia Liu, Shuo Wang, Yong Yang, Peng Du \\ Key Laboratory of Carcinogenesis and Translational Research (Ministry of Education), Department of Urology, Peking University Cancer Hospital \\ $\&$ Institute, Beijing, China \\ Contributions: (I) Conception and design: P Du; (II) Administrative support: Y Yang; (III) Provision of study materials or patients: P Du, S Wang; (IV) \\ Collection and assembly of data: X Tang, Y Cao; (V) Data analysis and interpretation: X Tang, J Liu; (VI) Manuscript writing: All authors; (VII) Final \\ approval of manuscript: All authors. \\ \#These authors contributed equally to this work. \\ Correspondence to: Peng Du. Key Laboratory of Carcinogenesis and Translational Research (Ministry of Education), Department of Urology, Peking \\ University Cancer Hospital \& Institute, No. 52 Fucheng Road, Haidian District, Beijing, China. Email: dupeng9000@126.com.
}

Backgrounds: This study aimed to evaluate the diagnostic and prognostic value of urine nuclear matrix protein 22 (NMP22) for bladder cancer.

Methods: A retrospective analysis was performed on 229 patients with bladder cancer who underwent transurethral resection of bladder tumor between 2015 and 2018 in our hospital. The sensitivity of NMP22 was calculated to evaluate the diagnostic value of NMP22. Logistic regression analyses were applied to investigate the prognostic value of NMP22 for pathologic features in bladder cancer.

Results: The sensitivity of NMP22 for the detection of bladder cancer was $28.82 \%$, and the false negative rate was $71.18 \%$. The sensitivity of NMP22 for the detection of low-grade disease and high-grade disease were $11.11 \%$ and $38.51 \%$. NMP22 had significantly higher sensitivity for the detection of highgrade bladder cancer $(\mathrm{P}<0.001)$. The sensitivity of NMP22 for the detection of Ta, T1 and T2 disease were $20.78 \%, 50.85 \%$ and $25.00 \%$ respectively (Ta refers to noninvasive papillary carcinoma, T1 refers to tumor invades subepithelial connective tissue, T2 refers to tumor invades muscularis propria). NMP22 had significantly higher sensitivity for detection of $\mathrm{T} 1$ disease $(\mathrm{P}<0.001)$. Univariate and multivariate logistic regression analysis suggested NMP22 might be an independent prognostic factor for high-grade $(\mathrm{P}<0.001)$ and $\mathrm{T} 1$ disease $(\mathrm{P}<0.001)$ in patients with bladder cancer.

Conclusions: Although the sensitivity of NMP22 was significantly higher in the detection of T1 and highgrade bladder cancer, the false negative rate was high. Besides, the NMP22 might be a prognostic factor for high-grade and T1 bladder cancer, but considering the limitations of this study, further studies are needed.

Keywords: Nuclear matrix protein 22 (NMP22); bladder cancer; oncology outcomes; pathologic features

Submitted Apr 14, 2020. Accepted for publication Sep 17, 2020.

doi: $10.21037 /$ tcr-20-1824

View this article at: http://dx.doi.org/10.21037/tcr-20-1824

\section{Introduction}

According to the cancer statistics in 2016, bladder cancer, commonly referred to the bladder urothelial carcinoma, was the fourth common new diagnosed malignant tumor in male, with about 76,960 new cases every year in both sexes in United States (1). In the field of bladder cancer treatment, trans-urethral resection of bladder tumor (TURBT), total and partial cystectomy are still the most

^ ORCID: 0000-0002-2499-5706. 
important treatments for resectable disease. For unresectable bladder cancer, chemotherapy and radiotherapy are classical treatments, and the emergence of immunotherapy and targeted therapy in recent years provides more treatment options for bladder cancer that cannot be controlled by radiotherapy and chemotherapy $(2,3)$. Considering that the prognosis of unresectable bladder cancer is significantly worse than that of resectable bladder cancer (3), the early diagnosis of bladder cancer is an important topic in clinical.

In the early diagnosis of bladder cancer, in addition to the traditional cystoscopy and urine cytology, molecular biomarkers are also used more and more because of its noninvasive and easy to implement. The molecular biomarkers of bladder cancer, that is, the components with diagnostic value in the urine of patients with bladder cancer, include exfoliated tumor cells, proteins, genes, and tumor metabolites. The detection of these biomarkers can provide valuable information for the diagnosis and follow-up of bladder cancer. At present, there are many biomarkers for molecular diagnosis of bladder cancer. Some detect specific proteins in urine, such as bladder tumor antigen (BTA) test, nuclear matrix protein 22 (NMP22) test, Cytokeratin 8 and 18 fragments test; some detect DNA in urine, such as AssureMDx test; some detect mRNA in urine, such as Xpert Bladder Cancer test and CxBladder Detect test; some detect tumor associated cellular antigens or aneuploidy for chromsomes in urine sediment, such as ImmunoCyt test and UroVysion test (4). Among these examinations, NMP22 is one of the most widely used in clinical practice.

Nuclear matrix proteins (NMPs) was isolated by Berezney in 1974 (5). As a protein accounting for about $10 \%$ of all nuclear proteins, the NMPs built the nuclear matrix together with peripheral lamins and pore complexes, and played an important role in the DNA replication and transcription (6). In 1996, a urinary protein named NMP22 was isolated by Keesee (7). Later it was reported that in bladder cancer the malignant transitional cells contained up to 80 times higher concentration of NMP22 than normal transitional cells (8). In non-muscle invasive bladder cancer (NMIBC), NMP22 were positive in $71.8 \%$ of cases, while the cytology were positive in $42.8 \%$ of cases for comparison (8). NMP22 assay had gained United States Food and Drug Administration (FDA) approval as an aid in the initial diagnosis of bladder cancer, and had been applied in clinical for years.

With the increasing popular use of the NMP22 in clinical, the sensitivity and specificity of NMP22 had been verified by several studies within past few years (8-10), however, the prognostic value of NMP22 in bladder cancer had not been investigated yet. Considering this, we conducted this study evaluating the association between urine NMP22 and the pathologic features in patients with bladder cancer to find if NMP22 could be a prognostic factor in bladder cancer, meanwhile the diagnostic value of NMP22 for bladder cancer was studied as well. We present the following article in accordance with the REMARK reporting checklist (available at http://dx.doi.org/10.21037/ tcr-20-1824).

\section{Methods}

\section{Patients}

A retrospective analysis was conducted to identify patients with bladder cancer at Peking University Cancer Hospital between the year 2015 and 2018. These patients would be enrolled: (I) adult patients aged 18 years or older, male or female; (II) diagnosed with bladder cancer and had not been performed with any kind of surgery; (III) performed with TURBT; (IV) bladder urothelial carcinoma was confirmed by pathology report with complete grading and staging information; (V) with urine NMP22 assay report. The following patients will be excluded: (I) complicated with other urogenital diseases, including acute or chronic inflammation of the urinary system; (II) combined with tumors in other sites; (III) complete pathology reports are not available. The study was conducted in accordance with the Declaration of Helsinki (as revised in 2013). This study was approved by the Ethics Committee of Peking University Cancer Hospital (approval No. 150204) and informed consent was taken from all the patients.

\section{Assay methods}

The Alere NMP22 BladderCheck Test kit (Scarborough, Maine, USA.) was used to qualitatively detect the urine NMP22. This test used a lateral flow immunochromatographic strip encased in a plastic cartridge to detect NMP qualitatively in the patient's urine sample. The antibodies in the lateral flow immunochromatographic strip were monoclonal antibodies (MAbs) raised against nuclear mitotic apparatus protein (NuMA) extracted from a cervical cancer cell line by the method of Fey and Penman (11). Two different MAbs were used, one as a capture antibody 
Table 1 Patients' clinicopathologic characteristics

\begin{tabular}{lc}
\hline Characteristics & Values \\
\hline Patients, $\mathrm{n}(\%)$ & $229(100.0)$ \\
Age (years), median (IQR) & $65(56$ to 71$)$ \\
Gender, $\mathrm{n}(\%)$ & $50(21.83)$ \\
Female & $179(78.17)$ \\
Male & \\
Pathologic grade, $\mathrm{n}(\%)$ & $81(35.37)$ \\
Low grade & $148(64.63)$ \\
High grade & \\
Pathologic stage, $\mathrm{n}(\%)$ & $154(67.25)$ \\
Ta & $59(25.76)$ \\
T1 & $16(6.99)$ \\
T2 & \\
NMP22, $\mathrm{n}(\%)$ & $163(71.18)$ \\
Negative & $66(28.82)$ \\
Positive &
\end{tabular}

IQR, interquartile range; NMP22, nuclear matrix protein 22.

and one as a reporter antibody. All samples were processed according to the instruction provided by the manufacturer. The voided urine samples were collected in plastic urine container and tested within 2 hours under room temperature. According to the written instruction and previous studies (10), a urine NMP22 level of 10 units/mL or above was considered to be positive.

\section{Study design}

Clinical characteristics data collected included patients medical record number, age, gender, NMP22 assay results. The pathologic features included histological tumor grade and pathologic tumor stage ( $\mathrm{T}$ stage). A single voided urine sample was collected prior to TURBT in all patients. The pathologic staging and histological grading of the bladder cancer were based on the American Joint Committee TNM Staging System for Bladder Cancer (7th edition, 2010) (12). Ta refers to noninvasive papillary carcinoma, T1 refers to tumor invades subepithelial connective tissue, T2 refers to tumor invades muscularis propria. All these data were obtained through a review of our hospital's electronic medical record.

\section{Statistical analysis methods}

Descriptive statistics were used to summarize patients' characteristics. Categorical variables are presented as numbers and percentages, continuous variables are presented as median and interquartile range (IQR). Patients were divided into two groups by the NMP22 results, NMP22 negative group and NMP22 positive group. The $t$-test was used to compare the continuous variables between these two groups. Chi-square test and crosstabs test were used to compare the categorical variables between these two groups. Then patients were divided into two groups by the pathologic grade results. The $t$-test was used to compare the continuous variables between patients with low grade bladder cancer and those with high grade disease. Chisquare test and crosstabs test were used to compare the categorical variables between these groups. Later all the patients were divided into three groups by the pathologic $\mathrm{T}$ stage (Ta, T1 and T2 group). The One-way ANOVA was used to compare the continuous variables between these three groups. Chi-square test and crosstabs test were used to compare the categorical variables between these groups. The logistic regression analysis was performed to determine the potential prognostic factor for pathologic outcomes in patients with bladder cancer using univariate and multivariate analysis. Statistical analysis was performed using Stata 14 for windows (StataCorp, College Station, TX, USA). All tests were two-sided and a value of $\mathrm{P}<0.05$ was considered statistically significant.

\section{Results}

\section{Data}

Patients' clinicopathologic characteristics were shown in Table 1. Two hundred and twenty-nine patients with bladder cancer were enrolled in our analysis from September 2014 through December 2018, including 81 (35.37\%) patients with low-grade disease and 148 (64.63\%) with high-grade disease. The median age was 65 years. There were 50 $(21.83 \%)$ female patients and $179(78.17 \%)$ male patients. Most patients had Ta $(\mathrm{n}=154)$ or T1 diseases $(\mathrm{n}=59)$, and 16 patients $(6.99 \%)$ were diagnosed with $\mathrm{T} 2$ disease.

\section{Analysis and presentation}

The sensitivity of NMP22 was shown in Table 2 and Figure 1. The sensitivity of NMP22 for the detection of bladder 
Table 2 Pathologic features according to NMP22

\begin{tabular}{lccc}
\hline \multirow{2}{*}{ Characteristics } & \multicolumn{2}{c}{ NMP22 } & \multirow{2}{*}{ P value } \\
\cline { 2 - 3 } & Negative & Positive & \\
\hline Patients, n (\%) & $163(71.18)$ & $66(28.82)$ & \\
Age (years), median (IQR) & $64(56$ to 69) & $67(58$ to 75$)$ & 0.095 \\
Gender, n (\%) & & & 0.618 \\
Female & $37(74.00)$ & $13(26.00)$ & \\
Male & $126(70.39)$ & $53(29.61)$ & \\
Pathologic grade, n (\%) & & & \\
Low-grade & $72(88.89)$ & $9(11.11)$ & \\
High-grade & $91(61.49)$ & $57(38.51)$ & \\
Pathologic stage, n (\%) & & & \\
Ta & $122(79.22)$ & $32(20.78)$ & \\
T1 & $29(49.15)$ & $30(50.85)$ & \\
T2 & $12(75.00)$ & $4(25.00)$ & \\
\hline
\end{tabular}

IQR, interquartile range; NMP22, nuclear matrix protein 22.

cancer was $28.82 \%$, and the false negative rate was $71.18 \%$. No significant difference could be detected regarding age between NMP22 positive patients and NMP22 negative patients $(\mathrm{P}=0.095)$. There was no significance in the distribution of gender between these two groups $(\mathrm{P}=0.618)$. The sensitivity of NMP22 for the detection of low-grade disease and high-grade disease were $11.11 \%$ and $38.51 \%$. NMP22 had significantly higher sensitivity for the detection of high-grade bladder cancer $(\mathrm{P}<0.001)$. The sensitivity of NMP22 for the detection of Ta, T1 and T2 disease were $20.78 \%, 50.85 \%$ and $25.00 \%$ respectively. NMP22 had significantly higher sensitivity for the detection of T1 disease $(\mathrm{P}<0.001)$.

Univariate and multivariate logistic regression analysis for prognostic factors associated with pathologic tumor grading were presented in Table 3. Univariate and multivariate logistic regression analysis for prognostic factors associated with pathologic tumor grade were presented in Table 4 . Univariate analyses suggested age $(\mathrm{P}<0.001$, OR 1.06, 95\% CI: $1.03-1.09)$, gender $(\mathrm{P}=0.006$, OR $2.44,95 \%$ CI: $1.29-4.63)$ and NMP22 ( $\mathrm{P}<0.001$, OR 5.01, 95\% CI: $2.32-10.80)$ were statistically significantly associated with pathologic grade, and gender ( $\mathrm{P}=0.014$, OR $2.66,95 \%$ CI: 1.22-5.82), NMP22 ( $\mathrm{P}<0.001$, OR 3.16, 95\% CI: 1.74-5.75) were statistically significantly associated with pathologic stage. Multivariate analyses showed that age $(\mathrm{P}<0.001$,
OR 1.06, 95\% CI: 1.03-1.09), gender ( $\mathrm{P}=0.007$, OR 2.67, $95 \%$ CI: $1.31-5.44)$ and NMP22 ( $<<0.001$, OR 5.19, 95\% CI: $2.30-11.70)$ were statistically significantly associated with pathologic grade, while gender $(\mathrm{P}=0.016$, OR 2.69, 95\% CI: 1.20-6.01) and NMP22 ( $<<0.001$, OR 3.11, 95\% CI: 1.69-5.74) were statistically significantly associated with pathologic grade, suggesting that gender (male) and NMP22 (positive) might be independent prognostic factors for T1 bladder cancer. Univariate and multivariate analyses suggested age was not a prognostic factor for pathologic stage $(\mathrm{P}=0.216$ and $\mathrm{P}=0.433$ separately), while age (older), gender (male) and NMP22 (positive) might be independent prognostic factors for pathologic high-grade bladder cancer.

\section{Discussion}

So far, no examination is perfect in the early diagnosis of bladder cancer. Cystoscopy was the gold standard method, but it was invasive, expensive, and inconvenient. Cytology was relatively cheap and convenient, but the sensitivity for low grade disease was not enough (13). NMP22 as a cheap, convenient assay with enough sensitivity and specificity, was recommended for daily practice in some studies $(14,15)$. But in other studies, it's reported that its diagnostic performance was limited (16,17). According to Wang and colleagues' meta-analysis, the reported NMP22 sensitivity was extremely varied, among $5.56 \%$ to $100 \%$ (15). There were huge differences among conclusions of different studies as well. In this study, only 66 out of 229 patients with pathologic confirmed bladder cancer were with positive NMP22. The sensitivity was $28.82 \%$, and the false negative rate was $71.18 \%$. We thought this false negative rate was too high to recommend using NMP22 alone in the early diagnosis of bladder cancer.

The reason why there is such a large gap in the sensitivity of NMP22 detection is to start with its detection principle. NMPs are the non-chromatin network framework of the nucleus, which determine the morphology of the nucleus and organize the DNA into three-dimensional structure (18). They play an important role in the process of DNA replication, transcription, RNA processing, gene expression regulation and so on (19). NMPs are a kind of insoluble proteins, but they can be decomposed in the process of apoptosis and released into the surrounding environment. More than ten kinds of NMPs have been identified, some of which are tissue-specific and tumor specific. NMP22 is one of many nuclear matrix proteins, which is specific in urothelial cells. The content of NMP22 
A

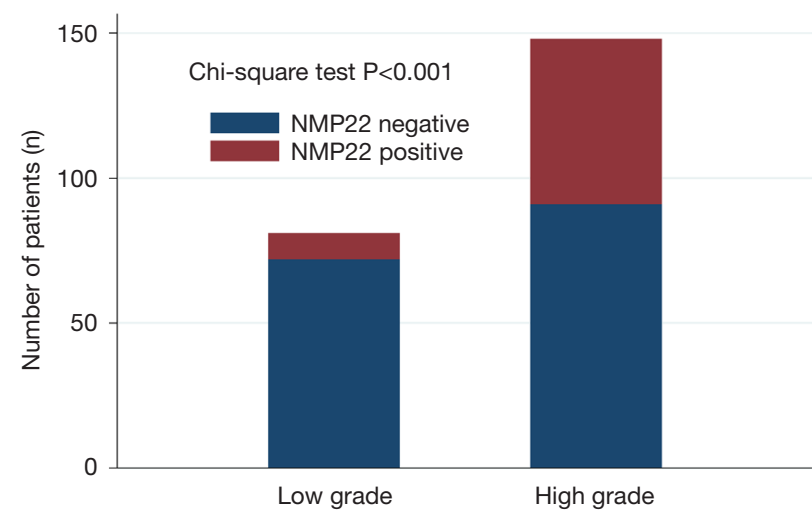

The sensitivity of NMP22 for the detection of low-grade and high-grade bladder cancer
B

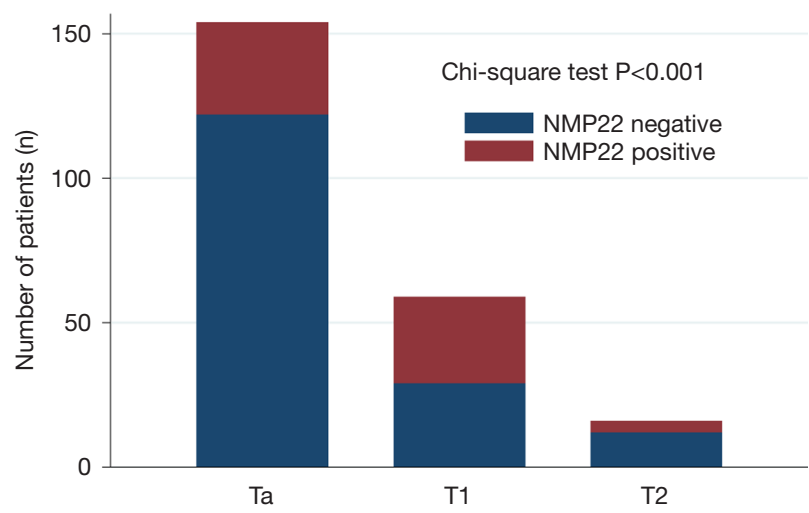

The sensitivity of NMP22 for the detection of Ta, T1 and T2 bladder cancer

Figure 1 Bar charts presenting the sensitivity of NMP22 for the detection of different diseases in bladder cancer. (A) The sensitivity of NMP22 for the detection of low-grade disease and high-grade disease were $11.11 \%$ and $38.51 \%$, and the corresponding false negative rate were $88.89 \%$ and $61.49 \%$. NMP22 had significantly higher sensitivity for the detection of high-grade bladder cancer $(\mathrm{P}<0.001)$. (B) The sensitivity of NMP22 for the detection of Ta, T1 and T2 disease were $20.78 \%, 50.85 \%$ and $25.00 \%$ respectively, and the corresponding false negative rate were $79.22 \%, 49.15 \%$ and $75.00 \%$ respectively. NMP22 had significantly higher sensitivity for the detection of T1 disease $(\mathrm{P}<0.001)$.

in cancerous urothelial cells is 80 times higher than that in normal cells (8). NMP22 in bladder cancer cells can be released into the urine in the form of cleavage fragments or complexes during cell apoptosis. The detection of these components in urine can help to determine whether there is bladder cancer or not. However, the disadvantages of this detection are also obvious. Due to the inconsistent rate of apoptosis and exfoliation of bladder cancer cells, the concentration of NMP22 released into urine will also change constantly. Therefore, the concentration of NMP22 in urine is not stable, but will change with time. For the same bladder cancer patient, the NMP22 concentration may be quite different between the first urination in the morning and the urine excreted after drinking a lot of water in the afternoon. Repeated tests may improve the detection rate, but the cost may be unacceptable to patients; at the same time, there is no relevant research on the relationship between specific detection times and detection rate. In addition, the test urine is taken by the patients themselves, so whether the patients keep the urine according to the doctor's instructions is uncertain, which may affect the experimental results as well. Current clinical studies have also found this phenomenon. In different studies (15), the sensitivity and specificity of NMP22 are quite different, which is also the reason why we believe that there is a risk of using NMP22 alone. However, this does not mean that NMP22 is worthless. During cystoscopy, some early bladder cancer or carcinoma in situ may be difficult to detect by naked eyes, but these tumor cells can release NMP22 into the urine. If combined with cystoscopy and NMP22 detection, the detection rate of bladder cancer could be significantly increased. Relevant studies have confirmed that, combined with NMP22 and cystoscopy, the detection rate of bladder cancer can be as high as 99\% (9). Therefore, it is the most important to fully understand the advantages and disadvantages of NMP22 test and reasonably apply it in clinical.

Considering the sensitivity of NMP22 might be different between different pathologic stages and grades. Jamshidian and colleagues conducted a research and reported the sensitivity of the NMP22 for Ta, T1, T2 disease were $31.3 \%, 90.0 \%$ and $66.7 \%$ respectively (20). In our study, the sensitivity of the NMP22 for Ta, T1 and T2 disease were $20.78 \%, 50.85 \%$ and $25.00 \%$ respectively. The NMP22 test had significantly higher sensitivity for the detection of $\mathrm{T} 1$ stage tumors $(\mathrm{P}<0.001)$. In terms of pathologic grade, In Jamshidian's study the sensitivity for grade 1, 2 and 3 disease were $66.7 \%, 81.8 \%$ and $84.6 \%$ respectively. In this study, the positive of NMP22 for low-grade and highgrade disease were $11.11 \%$ and $38.51 \%$. These results 


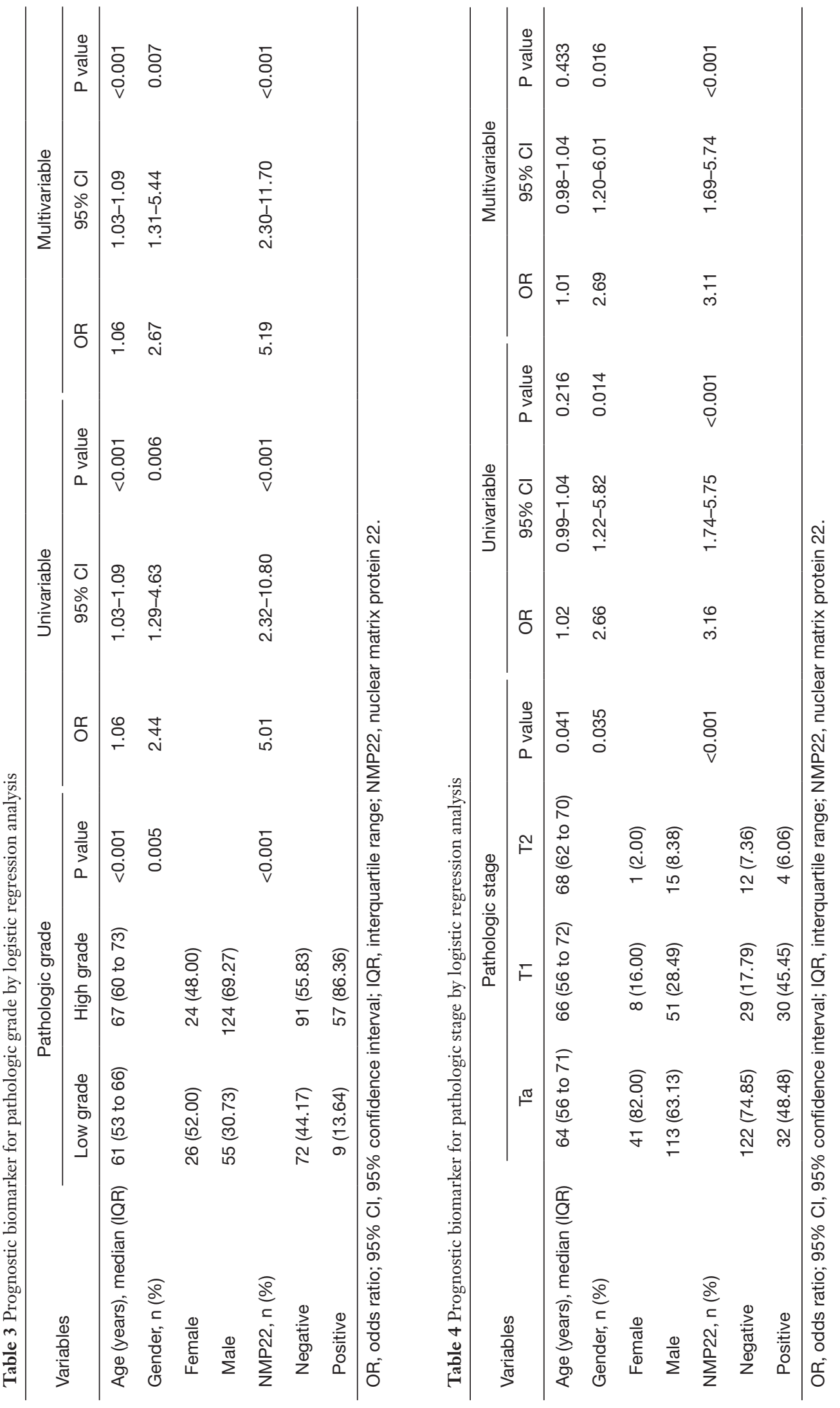


could not be compared directly since the grading systems were different, still we can find that the sensitivity of the NMP22 was significantly higher for the detection of highgrade disease $(\mathrm{P}<0.001)$ in our study. Wang and colleagues' pooled analyses had similar conclusion (15). Although the sensitivity of NMP22 was significantly higher in the detection of $\mathrm{T} 1$ and high-grade bladder cancer, the false negative rate was too high to recommend using NMP22 alone in the early diagnosis of bladder cancer, as missing the NMP22 negative tumor was dangerous to any patient.

Detection of NMP22 expression in tissues might increase the sensitivity of the test, but NMP22 assay is designed for noninvasive detection of bladder cancer, and this will lose the significance of NMP22 as a noninvasive test. In addition, if the tissue has been obtained, the diagnosis of bladder cancer can be made directly by pathological analysis, and NMP22 detection is not needed.

The expression of NMP22 in other tumors is related to the specificity of NMP22 detection. NMP22 is one of many nuclear matrix proteins, which specifically exists in urothelial cells. The content of NMP22 in cancerous urothelial cells is 80 times higher than that in normal cells (8). Therefore, NMP22 is designed for the detection of bladder cancer, and the research on NMP22 is almost all concentrated in the field of bladder cancer. We searched the relevant reports and found only two studies in other cancer $(21,22)$. These two studies analyzed the value of NMP22 in the diagnosis of renal cell carcinoma, and found that NMP22 might have diagnostic value for renal cell carcinoma, but there is no follow-up study.

Prognostic factors refer to the factors that can help to predict the prognosis of patients, usually predict the survival and recurrence of patients. In this study, we analyzed the predictive value of NMP22 in the pathological grading and staging of bladder cancer. That is to say, if NMP22 is positive, would the patient be more likely to be diagnosed with bladder cancer of a certain grade and stage. There are many prognostic factors in bladder cancer, including oncogene and tumor suppressor gene (Ras, ErbB, Rb, TP53, p21), cell proliferation and apoptosis related indicators (Ki-67, Fas, FasL), vascular endothelial growth factor (VEGF), epidermal growth factor (EGF), transforming growth factor (TGF), etc. These factors have certain value in predicting the survival and recurrence of bladder cancer (23). In terms of the prognostic value of NMP22 for bladder cancer, or the association of the NMP22 and the pathologic features of bladder cancer, we identified two previous studies. Zippe and colleagues (24) analyzed
18 patients with biopsy confirmed bladder cancer and 312 patients with benign disease of the bladder, and found there was no difference in NMP22 values when grade I and II cancers were compared with grade III cancers, and no significant difference between superficial ( $\mathrm{Ta} / \mathrm{Tis} / \mathrm{T} 1)$ versus invasive cancers (T2/T3). Jamshidian and colleagues (20) studied 76 patients with bladder cancer and 75 volunteers without bladder cancer, and found a significant association between the level of urine NMP22 and pathologic stage and grade of bladder cancer. In our study, logistic analysis revealed the NMP22 were independent prognostic factor for high-grade and T1 bladder cancer. Our conclusion was different from Zippe's conclusion, the possible explanation might be that only 18 patients with bladder cancer were enrolled in Zippe's study, and this sample size might be too limited to detect the significant difference. In Jamshidian's study, 75 patients were included, that was a larger sample size compared with that of Zippe's study, and the conclusion was similar with ours. In a word, we had the biggest sample size and the conclusion was that the NMP22 might be a prognostic factor for high-grade and T1 bladder cancer, and further studies were needed to clarify this conclusion.

However, some weakness could be identified in this study. This was a retrospective study with limited sample size, and the patients with T2 disease were not followed, that meant some patients underwent radical cystectomy might have different pathologic results latter. The survival was not analyzed in this study as well, because non-muscle invasive disease and muscle invasive disease should not be mixed in the same survival analysis. For patients with non-muscle invasive disease, the time is not long enough to detect the difference of survival between NMP22 negative patients and NMP22 positive patients. For patients with muscle invasive disease, 16 cases were too small a sample size to detect the significant difference of their survival. In addition, in this study, we did not analyze the specificity of NMP22 test in detecting bladder cancer, for only patients confirmed with bladder cancer were included. In this retrospective study, all patients were diagnosed with bladder cancer in outpatient, admitted to hospital for surgery, and confirmed as bladder cancer by postoperative pathology. These patients routinely completed NMP22 examination after admission. The sensitivity of NMP22 for bladder cancer was calculated by the formula: number of NMP22 positive bladder cancer patients/(number of NMP22 positive bladder cancer patients + number of NMP22 negative bladder cancer patients) $\times 100 \%$. The specificity formula was: number of NMP22 positive bladder cancer patients/(number of 
NMP22 positive bladder cancer patients + number of NMP22 positive healthy patients) $\times 100 \%$. Since we did not include any patients without diagnosis of bladder cancer, we did not analyze the specificity of NMP22 detection. Besides, some influencing factors were not taken into consideration in this study, for example the smoking status, body mass index (BMI), etc.

\section{Conclusions}

In conclusion, although the sensitivity of NMP22 was significantly higher in the detection of T1 and high-grade bladder cancer, the false negative rate was too high to recommend using NMP22 alone in the early diagnosis of bladder cancer. Besides, the NMP22 might be a prognostic factor for high-grade and T1 disease, but considering the limitations of this study, further studies are needed to clarify this conclusion.

\section{Acknowledgments}

Funding: The authors wish to thank the Capital's Funds for Health Improvement and Research (No. 2018-2-1025) and Beijing Natural Science Foundation (No. 7172044) for supporting the study.

\section{Footnote}

Reporting Checklist: The authors have completed the REMARK reporting checklist. Available at http://dx.doi. org/10.21037/tcr-20-1824

Data Sharing Statement: Available at http://dx.doi. org/10.21037/tcr-20-1824

Peer Review File: Available at http://dx.doi.org/10.21037/tcr20-1824

Conflicts of Interest: All authors have completed the ICMJE uniform disclosure form (available at http://dx.doi. org/10.21037/tcr-20-1824). The authors have no conflicts of interest to declare.

Ethical Statement: The authors are accountable for all aspects of the work in ensuring that questions related to the accuracy or integrity of any part of the work are appropriately investigated and resolved. The study was conducted in accordance with the Declaration of Helsinki (as revised in 2013). This study was approved by the Ethics Committee of Peking University Cancer Hospital (approval No. 150204) and informed consent was taken from all the patients.

Open Access Statement: This is an Open Access article distributed in accordance with the Creative Commons Attribution-NonCommercial-NoDerivs 4.0 International License (CC BY-NC-ND 4.0), which permits the noncommercial replication and distribution of the article with the strict proviso that no changes or edits are made and the original work is properly cited (including links to both the formal publication through the relevant DOI and the license). See: https://creativecommons.org/licenses/by-nc-nd/4.0/.

\section{References}

1. Siegel RL, Miller KD, Jemal A. Cancer statistics, 2016. CA Cancer J Clin 2016;66:7-30.

2. Babjuk M, Oosterlinck W, Sylvester R, et al. EAU Guidelines on Non-Muscle-Carcinoma of the Bladder. Actas Urol Esp 2009;33:361-71.

3. Guideline-Based Management of Muscle-Invasive Bladder Cancer: NCCN, ICUD, and EAU. New York: Springer, 2015. Available online: http://link.springer. com/10.1007/978-1-4939-1881-2_37

4. Oliveira MC, Caires HR, Oliveira MJ, et al. Urinary Biomarkers in Bladder Cancer: Where Do We Stand and Potential Role of Extracellular Vesicles. Cancers (Basel) 2020;12:1400.

5. Berezney R, Coffey DS. Identification of a nuclear protein matrix. Biochem Biophys Res Commun 1974;60:1410-7.

6. Getzenberg RH, Konety BR, Oeler TA, et al. Bladder cancer-associated nuclear matrix proteins. Cancer Res 1996;56:1690-4.

7. Keesee SK, Briggman JV, Thill G, et al. Utilization of nuclear matrix proteins for cancer diagnosis. Crit Rev Eukaryot Gene Expr 1996;6:189-214.

8. Kundal VK, Pandith AA, Hamid A, et al. Role of NMP22 Bladder Check Test in early detection of bladder cancer with recurrence. Asian Pac J Cancer Prev 2010;11:1279-82.

9. Grossman HB, Soloway M, Messing E, et al. Surveillance for recurrent bladder cancer using a point-of-care proteomic assay. JAMA 2006;295:299-305.

10. Giannopoulos A, Manousakas T, Gounari A, et al. Comparative evaluation of the diagnostic performance of the BTA stat test, NMP22 and urinary bladder cancer antigen for primary and recurrent bladder tumors. J Urol 
2001;166:470-5.

11. Fey EG, Penman S. Nuclear matrix proteins reflect cell type of origin in cultured human cells. Proc Natl Acad Sci U S A 1988;85:121-5.

12. Edge SB, Compton CC. The American Joint Committee on Cancer: the 7th edition of the AJCC cancer staging manual and the future of TNM. Ann Surg Oncol 2010;17:1471-4.

13. Kelly JD, Dudderidge TJ, Wollenschlaeger A, et al. Bladder cancer diagnosis and identification of clinically significant disease by combined urinary detection of $\mathrm{Mcm} 5$ and nuclear matrix protein 22. PLoS One 2012; 7:e40305.

14. Glas AS, Roos D, Deutekom M, et al. Tumor markers in the diagnosis of primary bladder cancer. A systematic review. J Urol 2003;169:1975-82.

15. Wang Z, Que H, Suo C, et al. Evaluation of the NMP22 BladderChek test for detecting bladder cancer: a systematic review and meta-analysis. Oncotarget 2017;8:100648-56.

16. Doğan C, Pelit ES, Yıldırım A, et al. The value of the NMP22 test for superficial bladder cancer diagnosis and follow-up. Turk J Urol 2013;39:137-42.

17. Casella R, Huber P, Blöchlinger A, et al. Urinary level of nuclear matrix protein 22 in the diagnosis of bladder

Cite this article as: Tang X, Cao Y, Liu J, Wang S, Yang Y, Du P. The diagnostic and prognostic value of nuclear matrix protein 22 in bladder cancer. Transl Cancer Res 2020;9(11):7174-7182. doi: $10.21037 /$ tcr-20-1824 cancer: experience with 130 patients with biopsy confirmed tumor. J Urol 2000;164:1926-8.

18. Berezney R. The nuclear matrix: a heuristic model for investigating genomic organization and function in the cell nucleus. J Cell Biochem 1991;47:109-23.

19. Konety BR, Getzenberg RH. Nuclear structural proteins as biomarkers of cancer. J Cell Biochem 1999;Suppl 3233:183-91.

20. Jamshidian H, Kor K, Djalali M. Urine concentration of nuclear matrix protein 22 for diagnosis of transitional cell carcinoma of bladder. Urol J 2008;5:243-7.

21. Huang S, Rhee E, Patel H, et al. Urinary NMP22 and renal cell carcinoma. Urology 2000;5 5:227-30.

22. Ozer G, Altinel M, Kocak B, et al. Value of urinary NMP-22 in patients with renal cell carcinoma. Urology 2002;60:593-7.

23. Solomon JP, Hansel DE. Prognostic factors in urothelial carcinoma of the bladder: histologic and molecular correlates. Adv Anat Pathol 2015;22:102-12.

24. Zippe C, Pandrangi L, Agarwal A. NMP22 is a sensitive, cost-effective test in patients at risk for bladder cancer. J Urol 1999;161:62-5. 